\title{
Interpretación de la regulación de flujos de agua a partir de un estudio de caso
}

\section{Interpretation of the regulation of water flows from a case study}

\section{Diego Alejandro Pérez Giraldo ${ }^{1}$}

Ingeniero ambiental, Magister universitario en eficiencia energética y cambio climático y Especialista en ingeniería hidráulica y ambiental

Investigador del grupo CIAB. Universidad Nacional Abierta y a Distancia

https://orcid.org/0000-0003-1869-6571

diego.perez@unad.edu.co

\section{Sandra Patricia Montenegro Gómez ${ }^{2}$}

Lic. Biología y Química, Esp. en Manejo y Conservación de Suelos y Aguas, Magister

en Ciencias Agraria-énfasis suelos, Dra. en Ciencias, área de concentración

Microbiología Agrícola.

Directora Centro de Investigación de Agricultura y Biotecnología.

Universidad Nacional Abierta y a Distancia - CIAB

https://orcid.org/0000-0003-0035-0089

sandra.montenegro@unad.edu.co

\section{Carlos Mario Duque Chaves 3}

Ingeniero ambiental, Magister en Desarrollo Sostenible y Medio Ambiente, Magister en Tecnología Ambiental

Investigador del grupo CIAB. Universidad Nacional Abierta y a Distancia

https://orcid.org/0000-0001-5433-5349

carlos.duque@unad.edu.co

\section{RESUMEN}

Los cambios en el manejo y planificación de las cuencas hidrográficas han condicionado la presencia de diferentes coberturas riparias, así mismo variación en las dinámicas de los cauces naturales, sumado a esto, la construcción de estructuras de protección longitudinales han llevado a una transformación de los cuerpos de agua, reduciendo el espacio de movilidad fluvial y disminución de conectividad lateral del cauce con sus riberas, rectificación de los cauces mediante cortes de meandros, entre otros factores que impactan negativamente el entorno natural y coloca en riesgo diversas formas de vida. El estudio de caso analizado, refleja la importancia de implementar herramientas preventivas de DOI: https://doi.org/10.22490/ECAPMA.2953 
desastres o problemáticas causadas por desequilibrio en la regulación de los flujos de agua.

Palabras clave: Ripario, cauce, meandros, fluvial.

\section{ABSTRACT}

The changes in the management and planning of watersheds have conditioned the presence of different riparian coverings, as well as variation in the dynamics of the natural channels, added to this, the construction of longitudinal protection structures have led to a transformation of the bodies of water, reducing the space of fluvial mobility and decrease of lateral connectivity of the channel with its banks; rectification of the channels through meander cuts, among other factors that negatively impact the natural environment and put at risk various forms of life. The case study analyzed reflects the importance of implementing preventive tools for disasters or problems caused by imbalance in the regulation of water flows.

Key words: Ripario, channel, meanders, fluvial.

\section{INTRODUCCIÓN}

La regulación de flujos hídricos es proporcionada por la cobertura y configuración del suelo. Es un servicio ecosistémico vulnerable por el grado de deforestación de las cuencas y la variabilidad climática en un contexto de incertidumbre debido a cambios globales (Núñez, Pinilla \& Pineda, 2015). La superficie ocupada por vegetación tanto arbórea como arbustiva, contribuye a la estabilización de márgenes y orillas de los cauces ya que aumentan considerablemente la resistencia a la erosión debida a la fuerza de la corriente (Smith, 1976, Vizcaíno, 2003) y propicia la existencia de zonas con una capacidad de retención de sedimentos muy elevada. 
La regulación de flujos hídricos hace parte de los servicios ecosistémicos de regulación a menudo invisibles $y$, que en la mayoría de los casos se dan por sentados. Cuando estos servicios se deterioran, las pérdidas son difíciles de recuperar (FAO, 2018). Los servicios hidrológicos incluyen la regulación de caudales para mitigar inundaciones, la recarga de acuíferos que mantienen caudales durante la época seca, la purificación del agua y el control de la erosión (MEA, 2005; Corredor, Fonseca \& Páez, 2012).

Por su parte la agricultura y la ganadería si bien son unos de los pilares de la economía mundial, han venido impactando de forma desfavorable los ciclos hidrológicos e hidrogeológicos. Lo anterior, viene definido en un sinnúmero de situaciones en las cuales los índices de escasez del recurso enmarcan el quehacer de algunas comunidades tanto desde la oferta, como desde la demanda.

El desarrollo de nuevas tecnologías de la información e interpretación de los datos, han permitido modelar y generar escenarios futuros relacionados con la oferta del recurso hídrico, incluyendo un nuevo concepto hidráulico y ambiental denominado restauración de ríos.

La restauración de ríos no es una técnica, sino quizá un derecho que tiene el cuerpo de agua a encauzar sus aguas de acuerdo con sus características y no a las que el hombre le imponga, es allí donde se puede encontrar un dificultad a la hora de definir restauración, pues a veces lo confundimos con rehabilitación, que es más un proceso inducido por el hombre, más no por el mismo entorno natural, como lo expresaron Karr \& Morishita (2001): "durante casi 200 años la transformación de los ríos ha sido sinónimo de progreso, sin embargo las condiciones sanitarias y ambientales actuales parecieran demostrar todo lo contrario". 
En Colombia existe el plan nacional de restauración, el cual nos brinda suficientes herramientas que proyecten un fortalecimiento para el recurso hídrico (plan nacional de restauración, 2015). Desde el campo científico, se han planteado algunas propuestas de restauración relevantes de restauración fluvial entre ellas la planteada por Ramirez (2015) a partir de un caso de estudio en la Quebrada Olivares-Minitas en el municipio de Manizales (Departamento Caldas) en Colombia, donde se propusieron los estudios necesarios para completar la valoración ambiental y acciones básicas para la restauración de la quebrada.

En lo que respecta a la regulación del flujo hídrico, el manejo de las áreas riparias debe propender por mantener bosques desarrollados de alta productividad lo cual permitiría regular el exceso hídrico durante los periodos lluviosos y mantener un mayor volumen y continuidad durante los periodos secos (Núñez, Pinilla \& Pineda, 2015).

El presente documento a partir de un estudio de caso presenta una mirada general de la importancia e impacto de la regulación y restauración de las fuentes hídricas, sobre la conservación de los servicios brindados por los ecosistemas.

\section{Estudio de caso - río Totuí}

\section{Estado actual del tramo de estudio del río Totuí}

El tramo de estudio de longitud aproximada $0.32 \mathrm{Km}$, se encuentra ubicado en la cuenca media del río Totui, con un caudal medio de $2.760 \mathrm{~m}^{3} / \mathrm{s}$ y caudal mínimo de $0.116 \mathrm{~m}^{3} / \mathrm{s}$, así mismo, con un rendimiento hídrico año medio de 45.4 $\mathrm{L} / \mathrm{s}^{*} \mathrm{Km}^{2}$ y un rendimiento hídrico año seco $1.945 .4 \mathrm{~L} / \mathrm{s}^{*} \mathrm{Km}^{2}$ (POMCA río Risaralda, 2017). 
Este tramo presenta una fuerte presión antrópica caracterizada en su mayoría por la cercanía a un pequeño caserío. Las alteraciones que presenta esta sección del río se deben principalmente a:

1. La variación del cauce natural del río por la construcción de estructuras de protección longitudinales, como gaviones.

2. El descenso del nivel de base del río debido a las extracciones para intervenciones antrópicas sobre el canal principal aguas abajo del sitio de actuación.

3. Corte de meandros o rectificación de alineamiento, lo que aumenta la velocidad del flujo y por ende su capacidad de socavación.

4. El cambio de usos en la llanura de inundación, por la invasión de la misma por cultivos y usos con fines de ganadería.

5. Vertimientos puntuales derivados de descargas residuales domésticas, caracterizados por la presencia de heces humanas y de animales.

\section{METODOLOGÍA ABORDADA}

En cuanto a la metodología utilizada, el análisis de la zona de estudio se realizó en dos etapas, la primera enmarcada dentro del análisis de campo, mediante el cálculo de variables hidrológicas y el análisis de la vegetación riparia, lo cual permitió determinar los patrones de intervención del tramo de estudio y sus afectaciones al medio, la segunda fase permitió mediante un análisis de de la información registrada en la etapa de campo lo cual permitió a travs análisis de los patrone hidrológicos identificar la problemática y determinar las actuaciones sobre el tramo evaluado. 


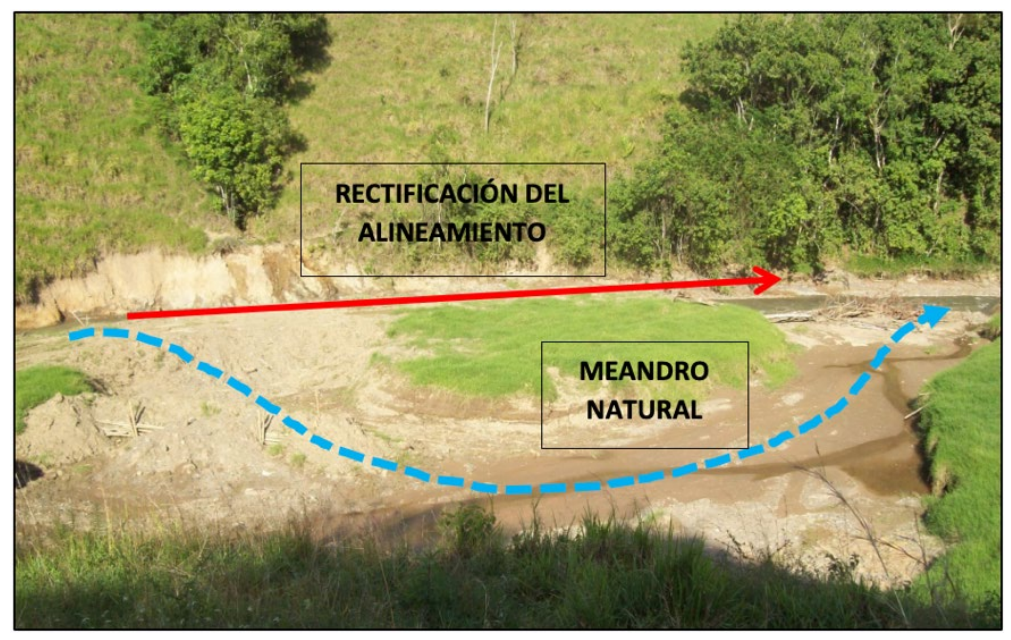

Figura 1. Afectación de meandros del rio Totui

Fuente: Autor.

\section{RESULTADOS PRELIMINARES Y DISCUSIÓN}

Rohde et al. (2006) describe el río como un agente natural que como tal debe estar debidamente estructurado, y como estructura debe seguir un estricto orden ecológico y socioeconómico que permita una interacción amigable del hombre con su ambiente natural y la dinámica fluvial. A continuación se describen los resultados obtenidos en las variables consideradas en el presente estudio.

\section{Perfil longitudinal}

El perfil longitudinal relaciona gráficamente la longitud del cauce con respecto a la altura sobre el nivel del mar (CAR, 2006). En el estudio del perfil longitudinal representado en la Figura 2 , se visualiza que se trata de un tramo de pendiente relativamente baja con un valor medio de $1.22 \%$ a lo largo del todo el trazado del río, y con una pendiente en el tramo de estudio del 1.5\%. Asimismo, presenta forma cóncava como corresponde al tramo medio de un río. 


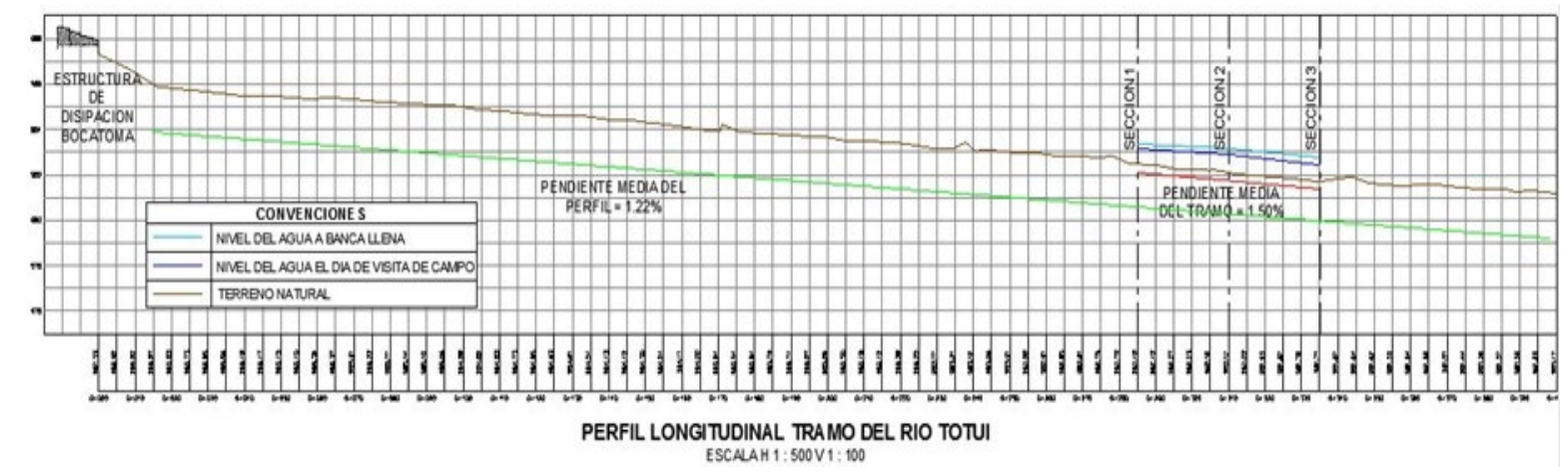

Figura 2. Perfil longitudinal del rio Totui. Fuente: Autor.

\section{Sinuosidad}

Del estudio de la sinuosidad, se obtiene un valor medio para la zona de actuación de 1.94 con lo cual podemos catalogar el río en esta sección como meándrico por presentar un $\mathrm{P}>1.5$, además y de acuerdo con la clasificación propuesta por Leopold \& Wolman (1957), este se puede clasificar como río meándrico con curvas "superficiales", puesto que este tipo de cauces cambian su curso en el transcurso del tiempo.

\section{Dinámica Fluvial}

El análisis realizado muestra una tendencia a la formación de barras puntuales, y procesos de cavitación, influenciado por la velocidad de la corriente, formando zonas de fuerte presión que origina burbujas que dan como resultado un desgaste de la roca y socavación de la misma.

Actualmente la zona de estudio cuenta con estructuras de protección longitudinales (gaviones), lo que podría disminuir el flujo entre la zona hiporreica y el canal natural, o afectar la rugosidad del cauce (n), disminuyendo en crecientes las posibilidades de que el río disipe su energía adecuadamente o no exista una recarga adecuada de los acuíferos subterráneos. 
Se observó en la zona de actuación, un uso del suelo con bosque mixto secundario, zonas cultivadas con caña de azúcar (Saccharum officinarum) y maracuyá (Passiflora edulisy), afectación alta por sobrepastoreo, con ausencia de material vegetal en zonas de ladera lo que propicia en épocas de lluvias fuertes avenidas de escorrentía, aumentando así el riesgo por sucesos catastróficos asociados a eventos de remoción en masa o aportación de materiales coluviales al río.

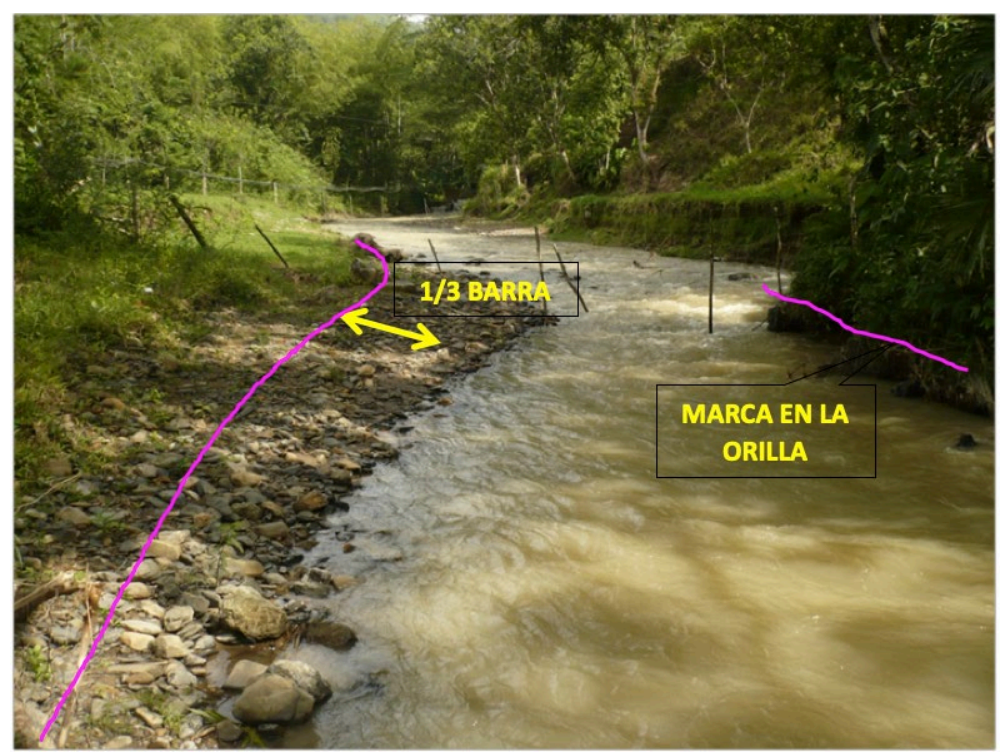

Figura 3. Indicadores de banca llena en barra y orilla. Fuente: Autor.

\section{Tipología de los problemas detectados}

- Impacto en la morfología fluvial: Espacio de movilidad fluvial limitado por obras de protección longitudinales (gaviones).

- Sustitución de vegetación de ribera: dominancia sobre la vegetación autóctona de cultivos de caña de azúcar (Saccharum officinarum) y maracuyá (Passiflora edulisy)

- Deforestación: Zonas deforestadas con fines de plantaciones comerciales o ganaderos. 


\section{Consecuencias de los problemas detectados}

presencia de obras de protección longitudinales (gaviones): Reducción del espacio de movilidad fluvial y disminución de conectividad lateral del cauce con sus riberas o zonas hiporreicas; rectificación de los cauces mediante cortes de meandros lo que traduce en un aumento de las velocidades y por ende de la socavación de orilla.

Cultivos de caña de azúcar y maracuyá: incompatibles con el desarrollo de la vegetación natural. Proliferación de especies alóctonas en detrimento de las autóctonas, y en consecuencia:

- Desestructuración de la vegetación de ribera.

- Desaparición de hábitats y nichos ecológicos.

- Contaminación con agroquímicos y residuos sólidos.

\section{Actuaciones para el mantenimiento y conservación del tramo de estudio del río Totui}

Las actuaciones corresponden a un conjunto de obras de acondicionamiento y conservación del Dominio Público Hidráulico cuya finalidad principal es la de facilitar la circulación de las aguas superficiales para evitar los problemas por inundaciones en las crecidas ordinarias de los ríos (Confederación hidrográfica del Duero, 2005). Entre los objetivos de las actuaciones se resalta la mejora de conectividad lateral del cauce con la ribera, aumentando el espacio de movilidad fluvial y recuperación de la composición y estructura de la vegetación de ribera, sustituyendo progresivamente las explotaciones comerciales por vegetación ripiara autóctona, por ejemplo, el árbol de Nogal (Juglans regia). 


\section{Líneas de actuación}

\section{Actuaciones para aumentar la anchura del espacio de movilidad fluvial:}

Construcción de obras de protección longitudinales (gaviones) en este caso para protección de los canales de aducción y desarenador, con materiales permeables que permitan un intercambio entre zonas. Se recomienda pues, la construcción de obras de bioingeniería (gaviones), dejando el revestimiento de la mismo situado en la base del talud sumergida por las aguas y las partes superiores libres para el crecimiento de la vegetación.

\section{Actuaciones para recuperar la vegetación de ribera:}

- Podas selectivas sobre las especies leñosas existentes para favorecer el correcto desarrollo del ecosistema

- Cortas finales y eliminación controlada de explotaciones productivas comerciales de caña de azúcar y maracuyá, escalonadamente, en varias campañas, según el turno tiempos de cada explotación, esto en zonas de protección de las márgenes de inundación del río.

- Reforestación y Forestación con especies autóctonas.

\section{Actuaciones para el saneamiento del río:}

- Control de agroquímicos mediante campañas de sensibilización y recogida de envases que contengan los mismos.

- Sistemas para el tratamiento de las aguas residuales domésticas debidamente diseñados y construidos (sistemas sépticos).

- Campañas de sensibilización para evitar que sean arrojados o depositados en las orillas del rio residuos sólidos.

\section{Conclusiones y consideraciones finales}


Aprovechar los recursos hídricos, es positivo siempre y cuando este aprovechamiento se realice de una manera sostenible, respetando cada una de las características morfológicas, geométricas y sanitarias que le permitan al río desarrollar libremente su dinámica, permitiendo aliviar su energía en los momentos adecuados y que regule naturalmente sus caudales, lo que permite el desarrollo de su vegetación riparia, la comunicación entre zonas laterales y húmedas, la formación de microorganismos controladores y por ende de comunidades de peces y pequeños mamíferos.

¿Qué se requiere en Colombia para una restauración ambiental fluvial? En primera instancia se requiere de un orden jurídico estricto, para que la restauración no continúe siendo vista como un mero hecho filosófico y paisajístico, sino como lo que verdaderamente es, permitiendo que los ríos se aproximen o vuelvan a su estado inicial y cumplan su función natural Palmer \& Allan (2006). En Colombia a partir de la observación de algunos hidrogramas de eventos torrenciales o de lluvia, es común detectar ausencia de laminación, pues ante la carencia de áreas inundables, sufre un aumento la curva de crecientes, esto quizás porque no existen unos usos adecuados del suelo o las políticas de ordenamiento territorial no son las adecuadas; por lo tanto, es prioritario dar un orden jurídico a muchas actuaciones.

Desde la perspectiva de implementación de herramientas que contribuyan con el uso del recurso hídrico de forma sostenible y sustentable, es importante considerar el indicador de Huella Hídrica, el cual permitirá proyectar el posicionamiento de los diferentes sectores, sociedad civil, empresas y gobierno, frente a esta temática, mostrando impactos asociados al desarrollo de las actividades económicas y sociales y sus implicaciones frente a la disponibilidad del recurso (Arévalo \& Sabogal, 2011). 


\section{BIBLIOGRAFÍA}

1. CAR (2006) Plan de ordenación y manejo de la cuenca del río magdalena. vertiente oriental departamento de Cundinamarca, Introducción.

2. Corredor Camargo, E., Fonseca Carreño, J., \& Páez Barón, E. (2012). Los servicios ecosistémicos de regulación: tendencias e impacto en el bienestar humano. Revista de Investigación Agraria y Ambiental, 3(1), 77-83. doi:https://doi.org/10.22490/21456453.936

3. FAO (2018) Servicios ecosistémicos y biodiversidad- Recuperado de http://www.fao.org/ecosystem-services-biodiversity/es/

4. Karr, J. R., \& Rossano, E. M. (2001). Applying public health lessons to protect river health. Ecology and Civil Engineering, 4(1), 3-18

5. MEA. 2005. Evaluación de Ecosistemas del Milenio. 2005. Ecosystems and human wellbeing: synthesis. Island Press, Washington, DC. Disponible en: www.millenniumassessment.org/

6. Palmer, M. A., \& Allan, J. D. (2006). Restoring rivers. Issues in Science and Technology, 22(2), 40-48

7. Rohde, S., Hostmann, M., Peter, A., \& Ewald, K. C. (2006). Room for rivers: An integrative search strategy for floodplain restoration. Landscape and Urban Planning, 78(1-2), 50-70

8. Vizcaíno, P., Magdaleno, F., Seves, A., Merino, S., del Tánago, M. G., \& de Jalón, D. G. (2003). Los cambios geomorfológicos del río Jarama como base para su restauración. Limnetica, 22(3-4), 1-8

9. Confederación hidrográfica del Duero (2005). Actuaciones de mantenimiento y conservación de cauces.

10. Ramírez Cardona, Jesica Leandra (2015) Propuesta metodológica para la valoración ambiental de corrientes hídricas desde la perspectiva de la restauración fluvial. Caso de estudio Quebrada Olivares-Minitas Manizales (Caldas). Maestría thesis, Universidad Nacional de Colombia Sede Manizales. 
11. Plan Nacional de Restauración (2015). Restauración Ecológica, Rehabilitación y Recuperación de Áreas Disturbadas. Ministerio de Ambiente y Desarrollo Sostenible. 92 p.

12. Arévalo, D., Lozano, J., \& Sabogal, J. (2011). Estudio nacional de huella hídrica Colombia sector agrícola. Revista Internacional de sostenibilidad, tecnología y humanismo, (6), 101-126.

13. Núñez, D. A. S., Pinilla, G. A., \& Pineda, J. E. M. (2015). Efectos del uso del suelo en las propiedades edáficas y la escorrentía superficial en una cuenca de la Orinoquia colombiana. Colombia forestal, 18(2), 255-27 14. CORPOCALDAS (2017). Plan de Ordenamiento y Manejo de la Cuenca del Río Risaralda. 